\title{
Up-to-downwave asymmetry of the CFOSAT SWIM fluctuation spectrum for wave direction ambiguity removal
}

\author{
Huimin Li, Danièle Hauser, Bertrand Chapron, Frédéric Nouguier, Patricia Schippers, Biao Zhang, Jingsong Yang \\ and Yijun $\mathrm{He}$
}

\begin{abstract}
The surface waves investigation and monitoring (SWIM) aboard the China-France Oceanography Satellite (CFOSAT), a pioneer conically scanning wave spectrometer, was successfully launched on October 29, 2018. Its innovative configuration composed of one nadir and 5 rotating near-nadir beams is designed to simultaneously observe the directional wave spectrum at global scale. In this study, we systematically implement the spectral analysis of the radar backscattering with the periodogram technique to obtain the fluctuation spectrum for each azimuth direction. The two-dimensional fluctuation spectrum of the three spectral beams $\left(\theta=6^{\circ}, 8^{\circ}, 10^{\circ}\right)$ combines all the azimuth directions within one entire rotation of $360^{\circ}$. The case study demonstrates that the wave features (peak wavelength and direction) are roughly consistent between the estimated fluctuation spectrum and the collocated WaveWatch III wave slope spectrum. A marked up-to-downwave asymmetry of the fluctuation spectrum with larger spectral level in the upwave direction for all the three spectral beams is observed. A ratio is defined between the fluctuation spectrum within the $\left[0^{\circ}, \mathbf{1 8 0}^{\circ}\right]$ sector relative to the $\left[180^{\circ}, 360^{\circ}\right]$ sector. Statistics display that this ratio is greater than 1 when it denotes the up-to-downave ratio and smaller than 1 for the down-to-upwave ratio. This observed spectrum asymmetry is linked to the asymmetric modulation from upwind to downwind. In addition, we employs such finding to help remove the $180^{\circ}$ wave direction ambiguity from a
\end{abstract}

This work is supported in part by the National Key Research and Development Program of China (under Grant 2016YFC1401002 and 2016YFC1401005), by Centre National d'études Spatiales (CNES) under the TOSCA program, by the National Natural Science Foundation (under Grant 42006163, 41620104003, 42027805 and 42076181), by the Joint Project between National Science Foundation of China and Russian Science Foundation (under Grant 42061134016), by the Startup Foundation for Introducing Talent of NUIST, by the European Space Agency and Ministry of Science and Technology Dragon 5 Cooperation Programme (ID. 58009).

Huimin $\mathrm{Li}$ is with School of Marine Sciences, Nanjing University of Information Science and Technology, Nanjing 210044, China and State Key Laboratory of Satellite Ocean Environment Dynamics, Second Institute of Oceanography, Ministry of Natural Resources, Hangzhou 310012, China. (E-mail: huimin.li@nuist.edu.cn).

Danièle Hauser is with LATMOS, Université Paris-Saclay, UVSQ, CNRS, Sorbonne Université, 78280 Guyancourt, France (e-mail: daniele.hauser@latmos.ipsl.fr).

Bertrand Chapron and Frédéric Nouguier are with IFREMER, Univ. Brest, CNRS, IRD, Laboratoire d'Ocánographie Physique et Spatiale (LOPS), Plouzane 29280, France (E-mail: bertrand.chapron@ifremer.fr; frederic.nouguier@ifremer.fr).

Biao Zhang and Yijun He are with School of Marine Sciences, Nanjing University of Information Science and Technology, Nanjing 210044, China. (E-mail: zhangbiao@nuist.edu.cn; yjhe@ nuist.edu.cn).

Patricia Schippers is with the ACRI-ST company, Guyancourt, France (Email: patricia.schippers@latmos.ipsl.fr).

Jingsong Yang is with the State Key Laboratory of Satellite Ocean Environment Dynamics, Second Institute of Oceanography, Ministry of Natural Resources, Hangzhou 310012, China (Email: jsyang@ sio.org.cn ).

Manuscript received Xx xx, 2020; revised xx xx, 2020 practical point of view. Preliminary results of the direction ambiguity removal displays a bias of $41.3^{\circ}, 40.6^{\circ}$ and $36.7^{\circ}$ for the beams, respectively. The $10^{\circ}$ beam shows slightly better performance compared to the other two beams in terms of the bias and standard deviation. This shall lay strong basis for the operational implementation of such algorithm to resolve the direction ambiguity.

Index Terms-CFOSAT SWIM; Up-to-downwave asymmetry of fluctuation spectrum; Wave direction ambiguity removal

\section{INTRODUCTION}

$\mathbf{O}$ CEAN waves are important processes in the upper ocean to regulate the heat, momentum exchange of the airsea interaction [1]. Since the directional wave spectrum can fully characterize the sea state, its observations are essential for wave related studies. Until recently, in-situ buoys and the coastal radars, including high-frequency radars and the Xband marine radar have been the chief source of the wave spectra measurements [2], [3], [4]. Yet, these techniques are usually restricted by their limited spatial coverage. Despite spaceborne synthetic aperture radar (SAR) has been proven capable of measuring the winds and waves at the global scale [5], [6], [7], however, waves along the SAR flight direction are usually distorted and therefore cannot be well resolved [8], [9]. This nonlinear loss of information in SAR imaging limits its widespread applications [10], [11]. An innovative technique is therefore necessary to complement the existing manners for accurate and comprehensive observations of directional wave spectrum at the global scale.

Radars operating at near-nadir incidence angles rely on the wave slope induced variations in the returned signal to observe the ocean waves. A conically scanning antenna receives backsatter at various azimuth angles to resolve the two-dimensional wave field. Such concept of a wave spectrometer has been proposed for over 30 years [12]. Its capability to observe the directional wave spectrum was later evidenced by multiple airborne experiments [13], [14]. One of the two payloads aboard the China-France Oceanography Satellite (CFOSAT), surface wave investigation and monitoring (SWIM) is such a wave spectrometer. SWIM is to some extent advantageous compared to the SAR wave observations in that its measurements are not distorted and have the potential to provide the twodimensional wave spectrum. The instrument characteristics as well as the forward simulation of SWIM backscattering have been detailed to support the mission design [15], [16]. The 
successful launch of CFOSAT marks the first ever deployment of a conically scanning real aperture radar (RAR) in the space [17], [18]. This shall facilitate the investigation of global ocean waves, not only regarding the wave height, but also the wave directional spectra to characterize the complex sea state [18]. It is expected to help advance our understanding of windwave interactions at global scale [19] as well as to refine the accuracy of numerical modeling [20], [21].

SWIM operates at $\mathrm{Ku}$-band with the center radar frequency at $13.575 \mathrm{GHz}$ [16]. It has six beams with 1 nadir and 5 near-nadir incidence angles $\left(0^{\circ}, 2^{\circ}, 4^{\circ}, 6^{\circ}, 8^{\circ}, 10^{\circ}\right)$. The nadir beam is similar to a state-of-the-art altimeter with comparable measurement accuracy for both the significant wave height and the wind speed [18]. The five near-nadir beams sequentially illuminates the sea surface at different azimuth angles. Each beam employs a chirp signal with the bandwidth of $320 \mathrm{MHz}$ for range detection along given azimuth. Spectral analysis of the backscattering profile along the range is conducted to obtain the modulation spectrum in that azimuth. Inversion of the wave height spectrum in this azimuth direction follows. The two-dimensional wave spectra is then accordingly constructed by combining all azimuth directions (about 50) within one rotation across the entire $360^{\circ}$.

At such low incidence angle as SWIM does, radar backscattering of the sea surface is dominated by the quasi-specular reflection from the wave slope satisfying the specular condition [22]. The geometrical modulation is the dominant mechanism for the signal contrast modulation. It is due to the change of local incidence angle provoked by the tilt of the long wave slopes. Assuming that the mechanism is linear (true for small slopes) and the hydrodynamic modulation is negligible, the measured modulation spectrum is linearly related to the waves slope spectrum via the tilt modulation transfer function (MTF) [23]. Under the approximation of small curvature effects, the backscattering at near-nadir incidence is proportional to the wave slope probability density function (PDF), the tilt MTF, the derivative of backscattering, is then related to the wave slope PDF. Most of the simulation studies presumed the wave slope spectrum to be Gaussian and isotropic, producing the simulated polar-symmetric modulation spectrum [15]. However, it has been reported that the modulation spectrum derived from the airborne observations was actually not symmetric [12]. They speculated that this is possibly due to the nonisotropic nature of wave slopes PDF and/or the second-order hydrodynamic modulation. However, no statistical analysis was presented due to the limited number of aircraft measurements in that paper [12]. In the meanwhile, many studies have disclosed that the up-downwave wave slope follows a nonGaussian shape based on both the sun glitter and the near-nadir microwave radar measurements [24], [25], [26], [27]. In other words, the derivative of wave slope PDF differs from upwave (or upwind) to downwave (or downwind) direction. The MTF variation relative to the wave directions might contradict with previous results of polar-symmetric modulation spectrum. In fact, the tilt MTF estimated based on the Global Precipitation Mission data has demonstrated the azimuthal dependence of MTF with larger values in the upwave (or upwind) [28].

As a matter of fact, it is the fluctuation signal that is directly calculated from the backscattering profile rather than the modulation signal. The former is proportional to the latter taking into influence of both speckle noise and radar impulse responses account [29]. These two spectra are related through the radar impulse response spectrum as well as the speckle noise spectrum [12], [13]. In contrast to a fixed antenna configuration, the rotating beams of SWIM allow to observe the sea surface at various azimuth directions. The direct comparison of fluctuation spectrum between the upwave and downwave direction becomes therefore feasible. In this study, SWIM observations are collocated with the directional wave spectra from WaveWatch III (WW3), and the dominant direction of the WW3 spectra are used as reference in the following analysis. The parameterizations of wave generation and dissipation proposed in [30] are used in the WW3 model with the wind forcing from the European Centre for MediumRange Weather Forecasts (ECMWF) forecast winds. This model configuration has been demonstrated to work well for $H_{s}$ and swell partitions compared to other parameterization packages [31]. Up-to-downwave asymmetry of the fluctuation spectrum is documented through comparison of the values at peak pairs with $180^{\circ}$ ambiguity. Note that one of the unsolved issue in the present SWIM data processing chain is the removal of the wave direction ambiguity [18]. We then present the preliminary results for removing the wave direction ambiguity by taking advantage of this up-to-downwave asymmetry.

The rest of this paper is organized as follows. Section II details the SWIM data included in this study as well as the spectral analysis to obtain the fluctuation spectrum. The up-todownwave asymmetry of fluctuation spectrum is presented in Section III and the preliminary results for direction ambiguity removal is given in Section IV. Discussion and conclusion then follow in Section IV.

TABLE I

DETAILS OF SWIM OBSERVATION SWATH $\mathrm{R}_{\text {swath }}$ AND GROUND-RANGE RESOLUTION $\mathrm{L}_{r}$.

\begin{tabular}{|c|c|c|c|}
\hline $\mathrm{INC}^{\circ}{ }^{\circ}$ & $\mathrm{R}_{\text {swath }}[\mathrm{km}]$ & $\mathrm{N}_{r}$ & $\mathrm{~L}_{r}[\mathrm{~m}]$ \\
\hline 2 & 18.1 & 4 & 53.9 \\
\hline 4 & 36.3 & 4 & 27.0 \\
\hline 6 & 54.5 & 2 & 9.0 \\
\hline 8 & 72.9 & 3 & 10.1 \\
\hline 10 & 91.5 & 3 & 8.1 \\
\hline
\end{tabular}

\section{DATA AND METHOD}

In this section, we first present the SWIM observations used in this paper as well as the collocated ancillary data. The spectral analysis using the periodogram method to calculate the fluctuation spectrum for each azimuth direction then follows.

\section{A. SWIM observations and ancillary data}

SWIM is a pioneer Ku-band RAR developed based on the concept detailed in [23]. It measures the radar backscattering 

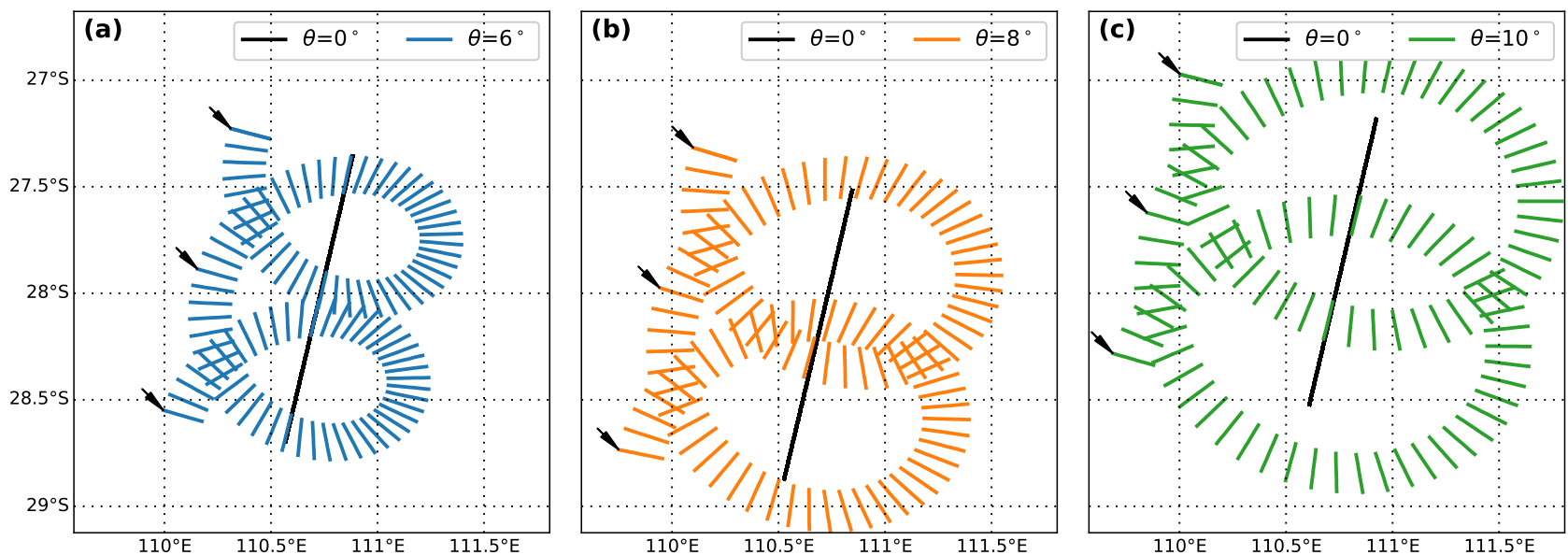

Fig. 1. An example for acquisition pattern of (a) $6^{\circ}$, (b) $8^{\circ}$ and (c) $10^{\circ}$ beams within two rotations of $360^{\circ}$. Black curve denotes footprint of the nadir beam. Note that the slightly varying coverage of these three incidence angles is due to their incontiguous azimuth directions at given moment. The black arrow indicates the starting position of one entire rotation.

with 6 beams at incidence angle from $0^{\circ}$ to $10^{\circ}$ with the increment of $2^{\circ}$. It is deployed in the orbit at an altitude about $519 \mathrm{~km}$. Radar footprint of the near-nadir beams is about $18 \mathrm{~km}$ by $18 \mathrm{~km}$ on the ground with the original slant range resolution of $0.47 \mathrm{~m}$. The spatial resolution and observation swaths in radius rely on the incidence angle. Details of the ground-range resolution and the observation swath are given in Table I. $N_{r}$ in the table gives the number of averaged range bins in the aboard processing. Since a certain number of range bins and azimuth samples needs to be averaged on board to reduce the downlink rate, the slant range resolution decreases between $0.9 \mathrm{~m}$ and $1.9 \mathrm{~m}$ depending on the number of averaged bins. More details of SWIM instrument parameters can be referred to [16].

Each of the near-nadir beams rotates at a speed of $5.6 \mathrm{rpm}$ to cover all azimuth angles with a directional sampling about $7.5^{\circ}$. It should be noted that at any given moment, the 5 near-nadir beams are not contiguous in azimuth angles due to the mechanical constraints in the rotating plate (see Figure 2 in [16]). The near-nadir beams of $6^{\circ}, 8^{\circ}, 10^{\circ}$ are dedicated to the measurements of directional wave spectra, which are often termed as spectral beams. The other two near-nadir beams of $2^{\circ}$ and $4^{\circ}$ are mainly kept to provide the backscattering profile relative to the incidence angle. We utilized the profile of calibrated and geocoded radar crosssection after instrument gain and geometry compensation for each spectral beam $\left(\theta=6^{\circ}, 8^{\circ}, 10^{\circ}\right)$. Such radar backscattering is annotated in the level-1A products. The modulation spectrum calculated by the French ground segment stored in the level-1B products are also included in this study for comparison with our computation. All the products are available upon registration via https://www.aviso.altimetry.fr/en/ missions/current-missions/cfosat/access-to-data.html. Fig. 1 presents an example of the acquisition pattern of the nadir and $6^{\circ}, 8^{\circ}, 10^{\circ}$ beams within two entire rotations. In this study, we define one rotation covering the entire $360^{\circ}$ to start from the azimuth direction closest to $270^{\circ}$ clockwise from the true North. Over one beam rotation about $10.8 \mathrm{~s}$, the characteristics of ocean waves being measured could be reasonably assumed to remain unchanged under low to moderate wind/wave conditions (about $<20 \mathrm{~m} / \mathrm{s}$ ). Therefore, all the azimuth directions within one rotation can then be combined to construct the two-dimensional fluctuation spectrum. Note that the validity of such hypothesis under tropical cyclones does not hold given the fast evolution of wave field over the spatial coverage of one entire rotation.

Numerous studies have demonstrated that at small incidence angle as SWIM operates, the radar backscattering is dominated by the quasi-specular reflection from the wave facets perpendicular to the radar line-of sight [22], [32]. According to [23], [33], the power spectrum of the modulated radar cross-section, the modulation spectrum $P_{M}(k, \phi)$ can then be written as

$$
P_{M}(k, \phi)=\frac{2 \pi}{L_{y}}\left(\cot \theta-\frac{\partial \ln \sigma_{0}}{\partial \theta}\right)^{2} k^{2} F(k, \phi)
$$

where $L_{y}$ is a length related to the azimuthal width of the beam footprint through [16]:

$$
L_{y}=\frac{R \beta_{a z}}{2 \sqrt{2 \ln 2}}
$$

where $R$ is the range distance and $\beta_{a z}$ the $3-\mathrm{dB}$ azimuthal bandwidth. $k$ and $\phi$ denote the wavenumber and azimuthal direction clockwise from the true North, respectively. $\theta$ is the radar incidence angle. $F(k, \phi)$ is the two-dimensional wave height spectrum and $\sigma^{0}$ is the SWIM-received normalized radar cross-section. As concluded in [12], the hydrodynamic modulation and nonlinear wave-wave interactions are solely of second-order effect and can be neglected at such nearnadir incidence angles. The modulation spectrum is associated with the fluctuation spectrum directly estimated from the backscattering signal by

$$
P_{F}(k, \phi)=P_{I R}(k) P_{M}(k, \phi)+P_{s p}(k)
$$

where $P_{I R}(k)$ is the impulse response spectrum and $P_{s p}(k)$ denotes the speckle spectrum. Note that this equation is an 
approximation by assuming that the radar signal is continuous and the impact of second-order noise term is negligible [16]. As annotated, these two spectra are usually assumed to be centro-symmetric across one rotation of $360^{\circ}$ [18]. This makes the up-to-downwave contrast of the fluctuation spectrum comparable to that of $P_{M}(k, \phi)$. Since this paper does not focus on inversion of the wave spectrum, up-todownwave asymmetry of only the fluctuation spectrum is investigated unless otherwise stated.

In this study, we utilized the SWIM observations acquired from 21 October to 5 November, 2019, which are processed with the updated on-board migration compensation algorithm [18]. The output of ocean wave spectral partitions from the WaveWath III (WW3) hindcast is collocated to be the reference wave direction. The original WW3 directional wave spectra are available at the spatial resolution of $0.5^{\circ}$ every 30 minutes. The WW3 wave spectrum is composed of 24 directions and 32 frequencies in the range of $0.037 \mathrm{~Hz}$ to $0.7 \mathrm{~Hz}$, covering the SWIM resolved wavelength range from $70 \mathrm{~m}$ to $500 \mathrm{~m}$. The partitioned peak wave period and peak wave directions are directly used here. All the collocated SWIM acquisitions are restricted within the latitude extent of $\pm 60^{\circ}$ in order to minimize the impact of sea ice on the WW3 wave spectrum. Reanalysis winds from the ECMWF are also included to examine the possible wind speed dependence of the spectral asymmetry, which are available at a spatial resolution of $0.25^{\circ}$ every 3 hours. Both WW3 and ECMWF products are collocated with the center of each SWIM rotation in terms of the nearest spatial and temporal distance.

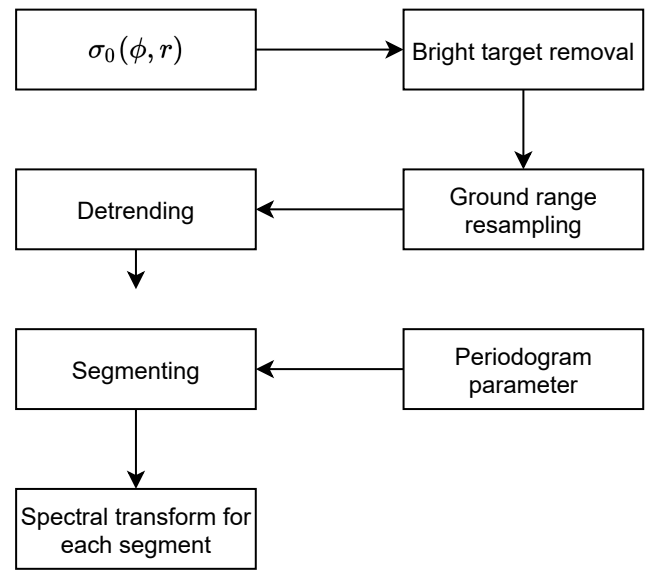

Fig. 2. Flowchart of the fluctuation spectrum estimate along each azimuth direction. The ground range resampling spacing is set to be $5 \mathrm{~m}$ and the periodogram window is 512 pixels with 256 pixels overlapping.

\section{B. Spectral analysis}

In this study, the spectral analysis for the three beams of $6^{\circ}, 8^{\circ}, 10^{\circ}$ is systematically carried out with the algorithm flowchart for the fluctuation spectrum at each azimuth given in Fig. 2. Since targets such as ships or platforms could cause very high backscattering signal, the pixels with $\sigma_{0}$ larger than 99\% percentile of that azimuth are replaced by the averaged value. Radar return is presented in the slant range geometry
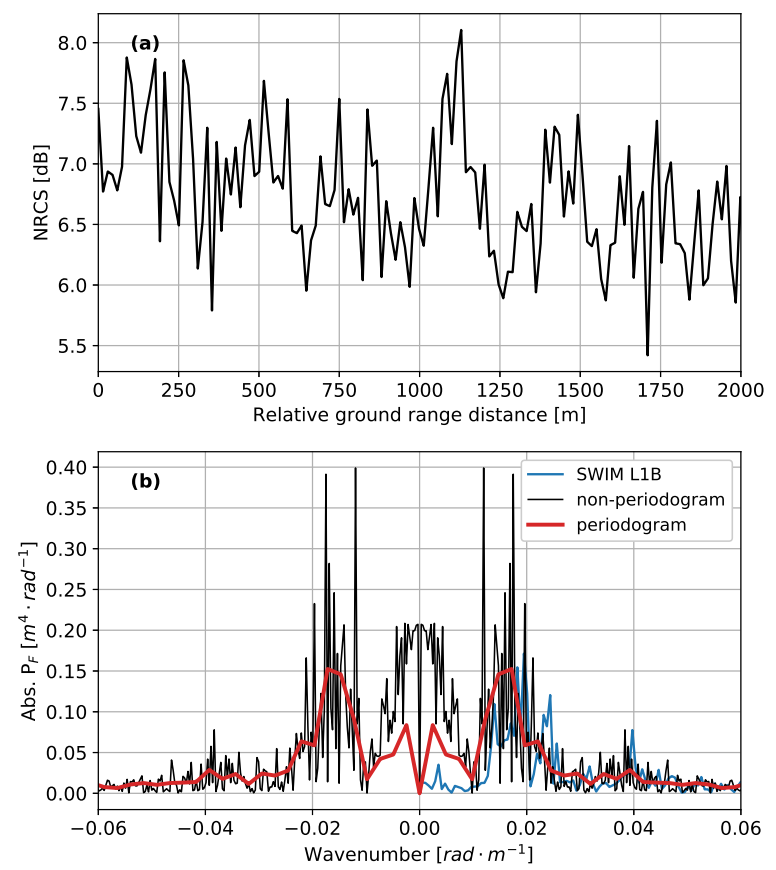

Fig. 3. An example for (a) SWIM measured $\sigma_{0}$ profile of $10^{\circ}$ beam with respect to the relative ground range distance; (b) Comparison of the obtained fluctuation spectrum using periodogram algorithm in Fig. 2 (red curve) and non-periodogram method (black curve) and the results annotated in SWIM level-1B products (blue curve).

with constant slant range spacing. But the slightly varying incidence angle from the near range to the far range results in an irregular ground range sampling. To facilitate the following spectral analysis, the radar signal is therefore resampled from the constant slant range spacing to the constant ground range spacing through the interpolation technique. Throughout this study, the ground range resampling is set to be $5 \mathrm{~m}$ for all three spectral beams. As shown in Fig. 3 (a), the radar return overall decrease from the near range towards the far range. We first estimate this decreasing trend with a lowpass Gaussian smoothing along the range. The detrending step in Fig. 2 subtracts this estimated trend from the radar backscattering profile and outputs the signal fluctuations $\delta \sigma_{0}$. In order to reduce the noise effect, the periodogram technique is employed. The signal fluctuations $\delta \sigma_{0}$ relative to the radial ground range is divided into segments at lengths of 512 pixels with half overlapping. This leads to 15 segments for each azimuth direction and one averaged spectrum is obtained in the end. Note that this algorithm differs from that used in the level2 processing chain by two aspects. On one hand, this algorithm takes advantage of the segments and periodogram to reduce speckle noise effect. On the other hand, we use the whole azimuthal sectors over $360^{\circ}$ including the ambiguous wave parts, rather than the standard boxes in the level-2 products which covering only $180^{\circ}$, to facilitate the following up-todownwave comparison.

An example of the $\sigma_{0}$ profile along the relative ground range distance is given in Fig. 3(a). A decreasing trend of $\sigma_{0}$ is evident, which is removed by the detrending step in Fig. 3 prior to the fluctuation spectrum estimate. The fluctuation spectra 

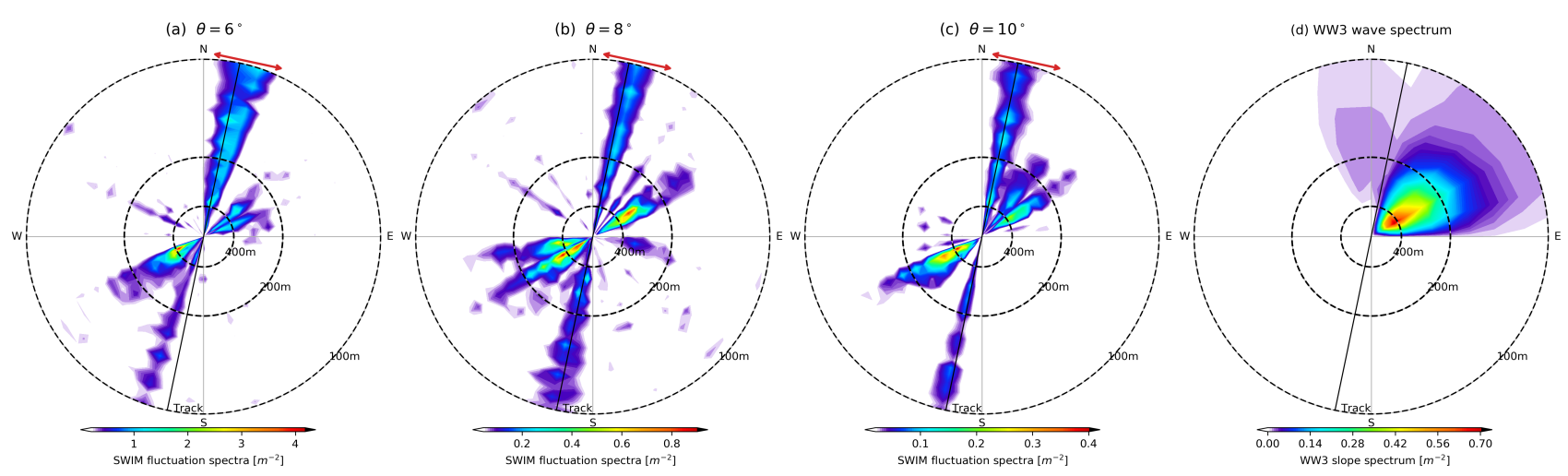

Fig. 4. Two-dimensional fluctuation spectrum constructed from one rotation of $360^{\circ}$ for the beam of (a) $6^{\circ}$, (b) $8^{\circ}$ and (c) $10^{\circ}$. The collocated WW3 wave slope spectrum is shown in (d) for comparison. The WW3 spectra direction corresponds to the direction that the waves travel to. The red arrow denotes the directional sector where the signal is strong affected by the instrument noise.

obtained using different methods are shown in Fig. 3(b). The thin black curve denotes the fluctuation spectrum calculated directly by the Fast Fourier Transform (FFT) of the $\sigma_{0}$ profile without zero-padding and averaging, which is quite noisy with high spectral signal at the low frequencies. For comparison, the fluctuation spectrum provided in the SWIM level-1b product is plotted as the blue curve. In this latter case, the FT was applied on the full-length segment (limit to the window where the signal-to-noise ratio is higher than $3 \mathrm{~dB}$ ), and the output spectrum is averaged over two consecutive wavenumbers. With our periodogram method as well as the averaged spectrum over multiple segments, the spectrum is less noisy and the signature is more clearer. Our estimated fluctuation spectrum is expected to provide reliable results. As such, we choose to compute the fluctuation spectrum along range direction at given azimuth angle using the periodogram method in the rest of this paper unless particularly stated. Note that in calculating the fluctuation spectrum, the resampled ground range spacing is $5 \mathrm{~m}$ and the periodogram size of 512 pixel is used, resulting in the wavenumber bin of $0.00245437 \mathrm{rad} \cdot \mathrm{m}^{-1}$.

\section{UP-TO-DOWNWAVE ASYMMETRY OF FLUCTUATION SPECTRUM}

In this section, a two-dimensional fluctuation spectrum computed using the periodogram method is first displayed and discussed as a representative example. The up-to-downwave asymmetry of the fluctuation spectrum between the two wave peaks with $180^{\circ}$ ambiguity is then presented from a statistical point of view.

\section{A. Case study}

The two-dimensional fluctuation spectrum is systematically constructed over one beam rotation of $360^{\circ}$. In general, there are approximately 50 azimuth directions within one rotation for each beam. Examples of the obtained directional fluctuation spectrum at positive wavenumbers are shown in Fig. 4 for the three spectral beams of $6^{\circ}, 8^{\circ}, 10^{\circ}$, respectively. The wind speed for this particular case is about $6.0 \mathrm{~m} / \mathrm{s}$. For each given azimuth angle, the spectral analysis of the radar fluctuation signal returns both negative and positive wavenumbers with symmetric magnitudes. In the construction of 2D fluctuation spectrum combining all the azimuth angles, only the positive wavenumber part are considered. Note that the angular sector within $\pm 15^{\circ}$ along the track direction is mostly contaminated by high speckle noise, which usually conceals the actual wave signal [18]. As such, this sector is simply masked out in the following analyses. The three beams display quite similar spectral signatures with one dominant wave system at wavelength around $400 \mathrm{~m}$. In the quantitative terms, the $6^{\circ}$ beam has the largest spectral magnitude, while the $10^{\circ}$ is the smallest. This decreasing spectral level might be partly associated with the impulse response function and the more significant contribution of speckle noise at $6^{\circ}$ due to fewer data points for the fluctuation spectrum estimate.

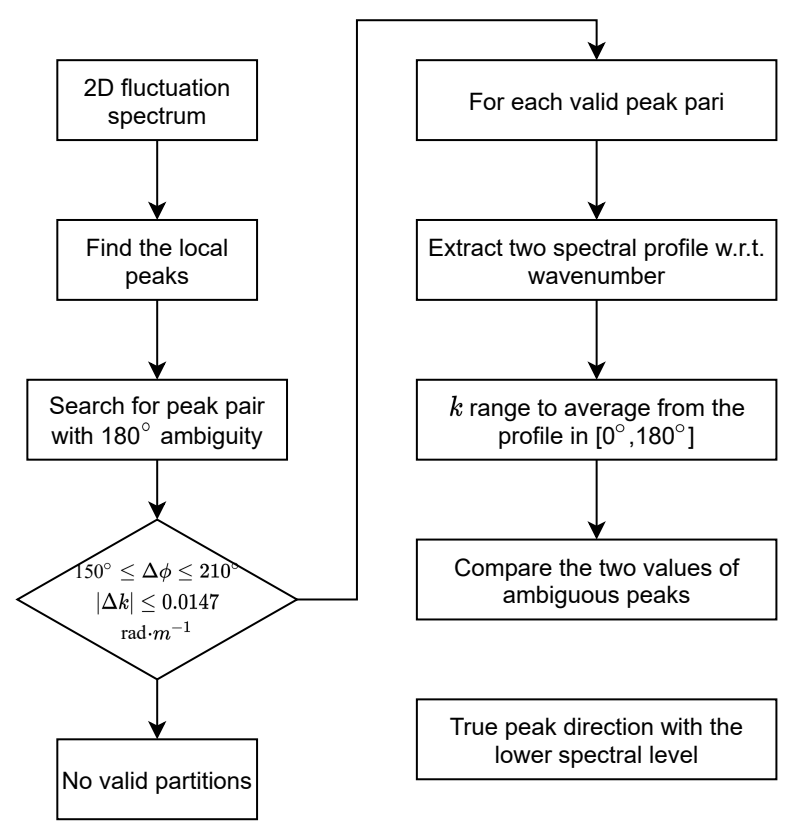

Fig. 5. Flowchart to search for the valid ambiguous peak pairs and to calculate the average of fluctuation spectrum around the identified peaks.

The peak directions of the fluctuation spectra shown in Fig. 4(a)-(c) are at $60^{\circ}$ and $240^{\circ}$ with the $180^{\circ}$ ambiguity. Since only the fluctuation spectral magnitude at positive 

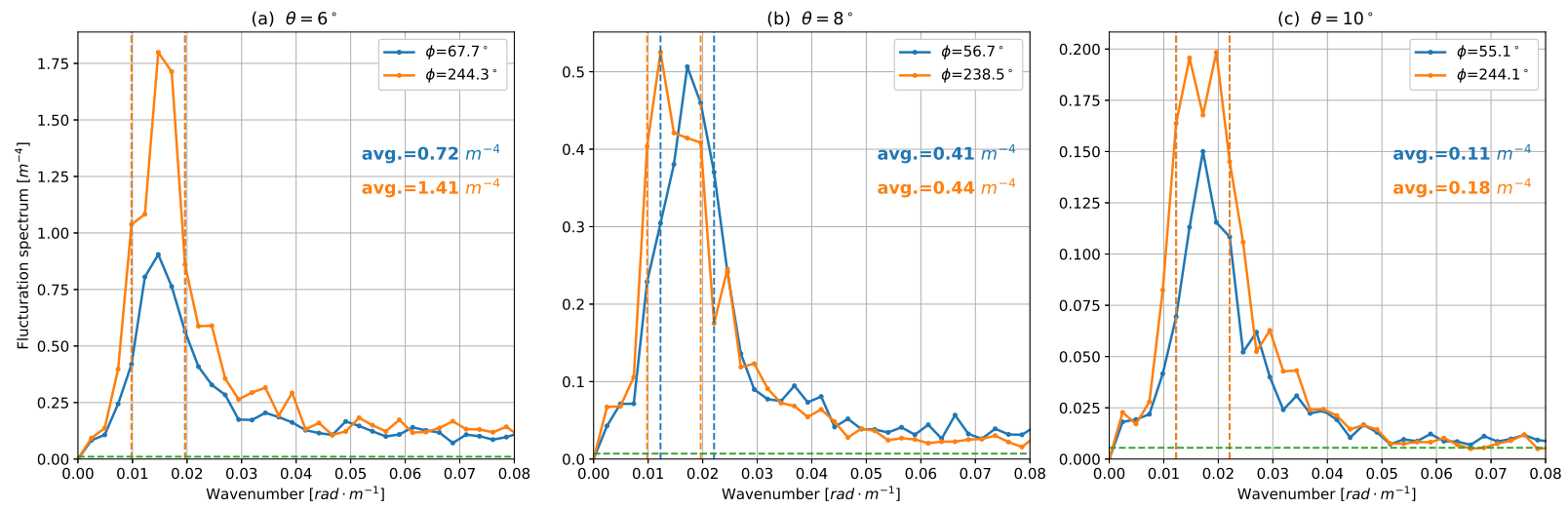

Fig. 6. Demonstration of the fluctuation spectral profile at the ambiguous peaks from the case shown in Fig. 4 for (a) $6^{\circ}$, (b) $8^{\circ}$ and (c) $10^{\circ}$ beams. The vertical dashed lines indicate the wavenumber range over which to calculate the averaged values. The averaged spectral magnitude is annotated in the same color with the curves. A constant for the speckle noise spectrum is reproduced based on [18] and plotted as the horizontal line in green, which is sufficiently small in comparison to the peak spectrum.

wavenumbers is included in the two-dimensional fluctuation spectrum, the magnitude is found smaller at the $60^{\circ}$ peak than the $240^{\circ}$ peak as shown. Such contrast is more evident for the $10^{\circ}$ beam in Fig. 4(c). The collocated WW3 wave slope spectra is shown in Fig. 4(d) to determine the true wave direction. In general, the peak wavelength is in good agreement with that of the fluctuation spectra for all three spectral beams about $400 \mathrm{~m}$. According to the WW3 wave spectra, the waves propagate towards the northeast direction. Thus, the spectral peak at $60^{\circ}$ in Fig.4(a)-(c) corresponds to the downwave part where the radar looking is in alignment with the wave traveling direction. While the peak at $240^{\circ}$ correspond to the upwave direction where the radar line-ofsight direction is opposite to the wave propagation direction. This case study demonstrates that the fluctuation spectra at downwave exhibits smaller magnitude than that at the upwave direction.

As a preparation to further statistics of the up-to-downave spectrum contrast, the spectral peak pairs with $180^{\circ}$ ambiguity are consistently detected. Fig. 5 presents the flowchart to search for the valid peak pairs and to compute the averaged fluctuation spectrum over the detected peaks. In this study, we employs the algorithm of finding local maxima in the Python programming language to identify local peaks of the two-dimensional fluctuation spectrum. The neighboring local maxima are merged if their indices of wavenumber bins are smaller than 5 . The peak pairs are determined with the wavelength difference of these two ambiguous parts smaller than $0.0147 \mathrm{rad} \cdot \mathrm{m}^{-1}$ and their direction difference within $180 \pm 30^{\circ}$. For each pair, the fluctuation spectra profile relative to the wavenumber is averaged along the line connecting one identified peak to the coordinate origin. Fig. 6 illustrates such extracted profiles from the fluctuation spectra shown in Fig. 4 for the three incidence angles. It is worth noting that the fluctuation spectral magnitude near the peaks is much higher than the speckle noise level reported in [18]. The speckle noise spectrum is shown to be wavenumber variant and exhibits slight variation at low wavenumber. For simplicity, we reproduced a speckle noise constant by averaging the speckle noise spectrum over all the azimuth direction within the wavenumber range of $\left[0.01 \mathrm{rad} \cdot \mathrm{m}^{-1}, \mathrm{rad} \cdot \mathrm{m}^{-1}\right]$ of the fluctuation spectrum [18], which is plotted as the green horizontal lines in Fig. 6. The wavenumber range over which to calculate the spectrum average is determined by the $3-\mathrm{dB}$ bandwidth of each profile. The spectral average over the selected wavenumber bandwidth is calculated rather than using solely the maximum values in order to minimize the uncertainty and accordingly increase the robustness of the proposed algorithm. This range is marked by the vertical dashed lines in the same color with the corresponding curves. As shown in Fig. 6, the averaged spectral signal around the direction of $60^{\circ}$ (blue curve) is smaller than that around $240^{\circ}$. In other words, the averaged spectrum at the downwave peak is lower in comparison to its ambiguous counterpart. In this case, the difference of averaged profile around the peaks within $\left[0^{\circ}, 180^{\circ}\right]$ and within $\left[180^{\circ}, 360^{\circ}\right]$ is negative as it represents the difference of downwave part relative to the upwave. Considering the magnitude of this difference relying on the absolute value of the fluctuation spectrum, it is not straightforward to reflect their statistical relativity. As an alternative, we define the ratio of spectral average over the peak $\phi_{1}$ in $\left[0^{\circ}, 180^{\circ}\right]$ to $\phi_{2}$ in $\left[180^{\circ}, 360^{\circ}\right]$, expressed as

$$
R T_{P}=\frac{\left\langle P_{F}\left(\phi_{1}\right)\right\rangle}{\left\langle P_{F}\left(\phi_{2}\right)\right\rangle}, \phi_{1} \in\left[0^{\circ}, 180^{\circ}\right], \phi_{2} \in\left[180^{\circ}, 360^{\circ}\right]
$$

$R T_{P}$ is so defined that it is independent of the absolute spectral level. $\langle\cdot\rangle$ represents the average of $P_{F}$ over the $3 \mathrm{~dB}$ wavenumber bandwidth as shown in Fig. 6. Here it is assumed that the peak spectral level is much higher than the speckle noise, which holds true for wave directions away from the track angle. $R T_{P}$ represents the down-to-upwave ratio of averaged fluctuation spectrum when the true wave direction (traveling to) is within $\left[0^{\circ}, 180^{\circ}\right]$. While it is the up-to-downwave ratio if the true wave direction falls within $\left[180^{\circ}, 360^{\circ}\right]$. The wave direction shown in Fig. 4 is about $60^{\circ}$ so that the $R T_{P}$ denotes the down-to-upwave ratio for this case. As demonstrated in Fig. $6, R T_{P}$ varies relative to the SWIM incidence angles, with values of 0.51 for $6^{\circ}$ beam, 0.93 for $8^{\circ}$ beam and 0.61 for $10^{\circ}$ beam. The fact that $R T_{P}$ 
is smaller than one illustrates that for this selected case, the fluctuation spectrum is smaller in the downwave direction than in the upwave direction.

\section{B. Statistics of fluctuation spectrum $R T_{P}$}
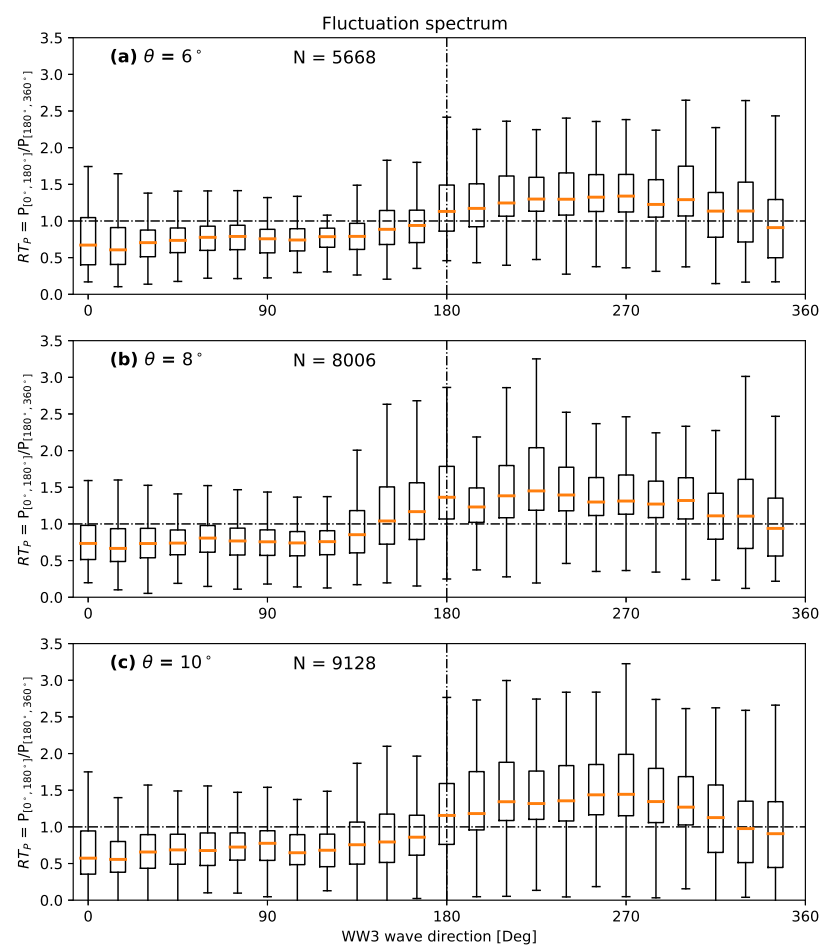

Fig. 7. Box plot of $R T_{P}$ as defined by Eq. 4 is given regarding the identified WW3 wave peak direction for (a) $6^{\circ}$, (b) $8^{\circ}$ and (c) $10^{\circ}$ beams. ' $N$ ' annotated in each plot denotes the number of valid peak pairs. The lower and higher end of the box are the first (25\%) and the third quartile (75\%), respectively. The horizontal line within the box represents the median value. Wave direction is relative to the true north $\left(0^{\circ}\right)$ in clockwise rotation. The wave direction bin is $15^{\circ}$.

Though the case in Fig. 4 has manifested the up-todownwave asymmetry of the fluctuation spectrum, it is not yet plausible to conclude its generality. In order to examine this asymmetry from a statistical point of view, the two ambiguous directions of fluctuation spectrum need to be categorized into the relative wave directions. In this study, we associated the identified spectral peak pairs with the WW3 wave peaks in terms of the spectral distance, which is defined as [34]

$$
S D=\frac{1}{60}\left(\left|D_{1}-D_{2}\right|+2 \times \frac{\left|T_{1}-T_{2}\right|}{T_{1}+T_{2}} \times 250\right)
$$

where $D_{1}, D_{2}$ are the detected SWIM and the WW3 peak direction, respectively. $T_{1}$ and $T_{2}$ are the peak wave period in the unit of $s$. By definition, $S D$ takes the impact of both wave period and the propagation direction into account to assess the similarity of two peaks. The value of 2 is added to describe errors in the wave period. While the factors of 60 and 250 are chosen to ensure that a $30^{\circ}$ difference in wave direction and $12 \%$ in wave period give a spectral distance of 1 . The peak pair is kept if either of the ambiguous peaks obtains the spectral distance, $S D$, smaller than 3 relative to its WW3 counterpart. There are initially 867, 1194, 1443 peak pairs identified based on the directional fluctuation spectrum. The criteria of spectral distance smaller than 3 leads to 647, 855, 975 pairs of $6^{\circ}, 8^{\circ}$ and $10^{\circ}$ beams for the statistical analyses, respectively. Most of the eliminated spectral peaks locate at wavelengths longer than $600 \mathrm{~m}$ (not shown), which is beyond the detection range of SWIM. This is because SWIM can only detect ocean wave in the range of [70 m,600 m] [18] and no peak pairs could be associated with the WW3 wave spectrum beyond this wavelength.

To examine the fluctuation spectrum asymmetry from a statistical point of view, the box plot of $R T_{P}$ as defined by Eq. (4) is shown in Fig. 7 relative to the collocated WW3 peak wave direction. Note that the speckle noise spectrum constant has been subtracted from the fluctuation spectrum to compute the $R T_{P}$. As annotated in Fig. 6, the constants are $0.0105 \mathrm{~m}^{-4}, 0.007 \mathrm{~m}^{-4}, 0.0055 \mathrm{~m}^{-4}$ for the $6^{\circ}, 8^{\circ}$ and $10^{\circ}$, respectively. The up-to-downwave asymmetry is prominent for all the three spectral beams, featured by the $R T_{P}$ either smaller or higher than 1 depending on the wave directions. When $\phi$ lies in the range of $\left[0^{\circ}, 180^{\circ}\right], R T_{P}$ characterizing the downto-upwave ratio is smaller than 1 , which further confirms the lower fluctuation spectra at downwave direction as observed in the case study. While in the case of wave direction belonging to $\left[180^{\circ}, 360^{\circ}\right], R T_{P}$ representing the up-to-downwave ratio is well above the horizontal line of 1 as marked by the dashed line. In consequence, it is reasonable to conclude that the fluctuation spectrum indeed exhibits a notable upto-downwave asymmetry with higher spectral levels at the upwave direction.

Note that the distribution of $R T_{P}$ slightly differs among the three spectral beams as shown in Fig. 6. Within the wave direction range of $\left[0^{\circ}, 180^{\circ}\right]$, the $10^{\circ}$ beam shows a much tighter distribution with its upper interquartile $(75 \%$ of the points) below the unit line. While for both $6^{\circ}$ and $8^{\circ}$, the $R T_{P}$ spans across the horizontal line of one for a couple of wave directions. By contrast, within the wave direction of $\left[180^{\circ}, 360^{\circ}\right]$, the lower interquartile $(25 \%)$ of all three spectral beams is mostly above the one line. The behavior that the $R T_{P}$ spanning on both sides of the one line is expected to introduce biases in applications based on this up-to-downwave asymmetry. In addition, numbers of the identified peak pairs are not the same for the three spectral beams. This is due to the fact that one peak pair is not consistently present in the fluctuation spectrum of the three beams. The refinement of fluctuation spectrum estimates or improvement in the level-1 radar signal shall be devised.

We also looked into the $R T_{P}$ dependence on the wind speeds, peak wavenumbers and wind directions as shown in Fig. 8. There is no clear variation of $R T_{P}$ relative to wind fields and the peak wavenumbers. The median value of $R T_{P}$ for each variable bin mostly distribute around the horizontal line of 1 as presented. In summary, we could conclude that the up-to-downwave asymmetry of the fluctuation spectrum is solely associated with the relative wave direction. This lays strong basis for the following attempt to remove the wave direction ambiguity by taking advantage of this up-todownwave asymmetry. It is worth noting that its independence on other environmental conditions facilitates the resolution of 

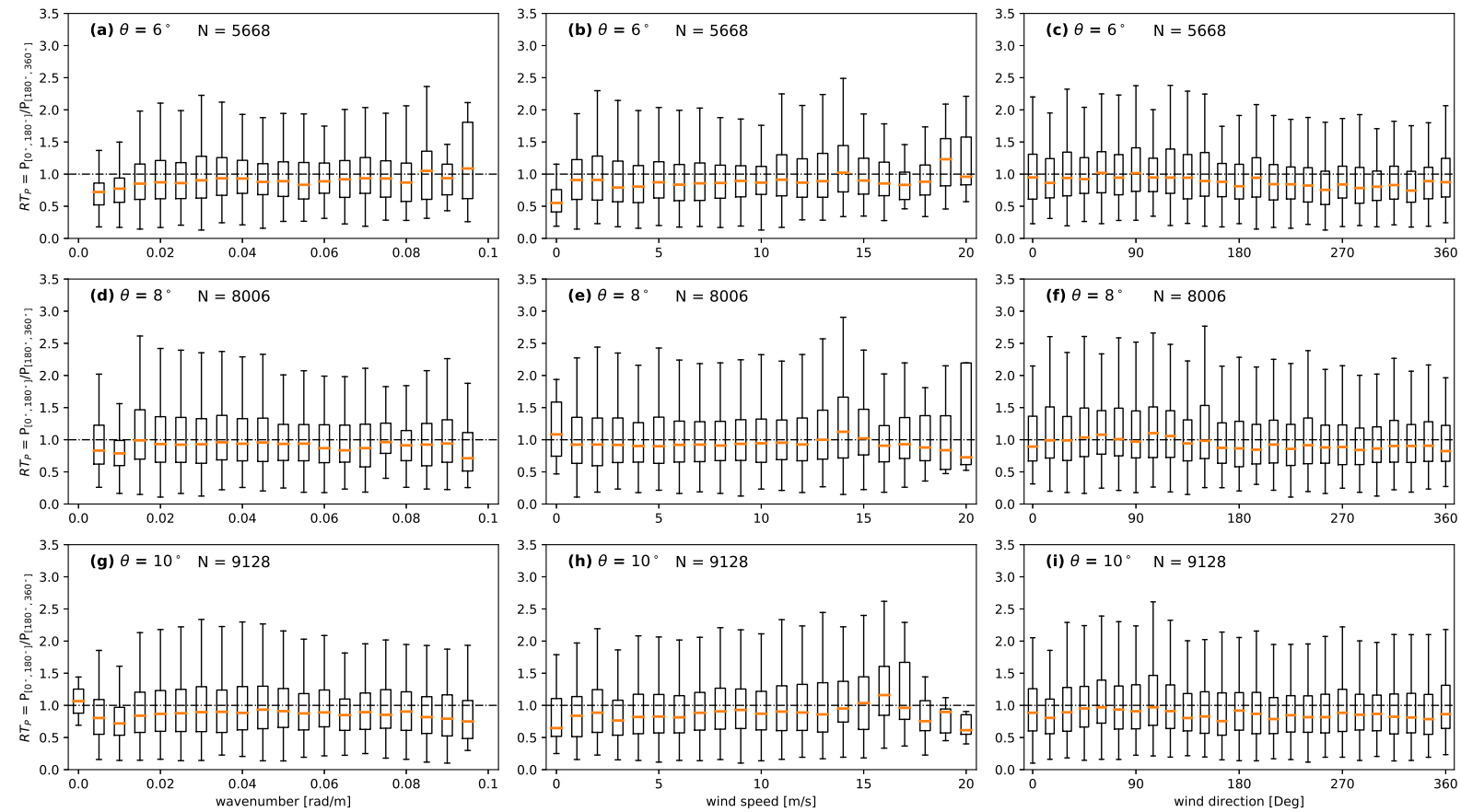

Fig. 8. Box plot of $R T_{P}$ as defined by Eq. 4 shown for $6^{\circ}, 8^{\circ}$ and $10^{\circ}$ beams relative to the WW3 wave peak wavenumber (a,d,g), wind speed (d,e,h) and wind direction (c,f,i), respectively. ' $\mathrm{N}$ ' annotated in each plot denotes the number of valid peak pairs.
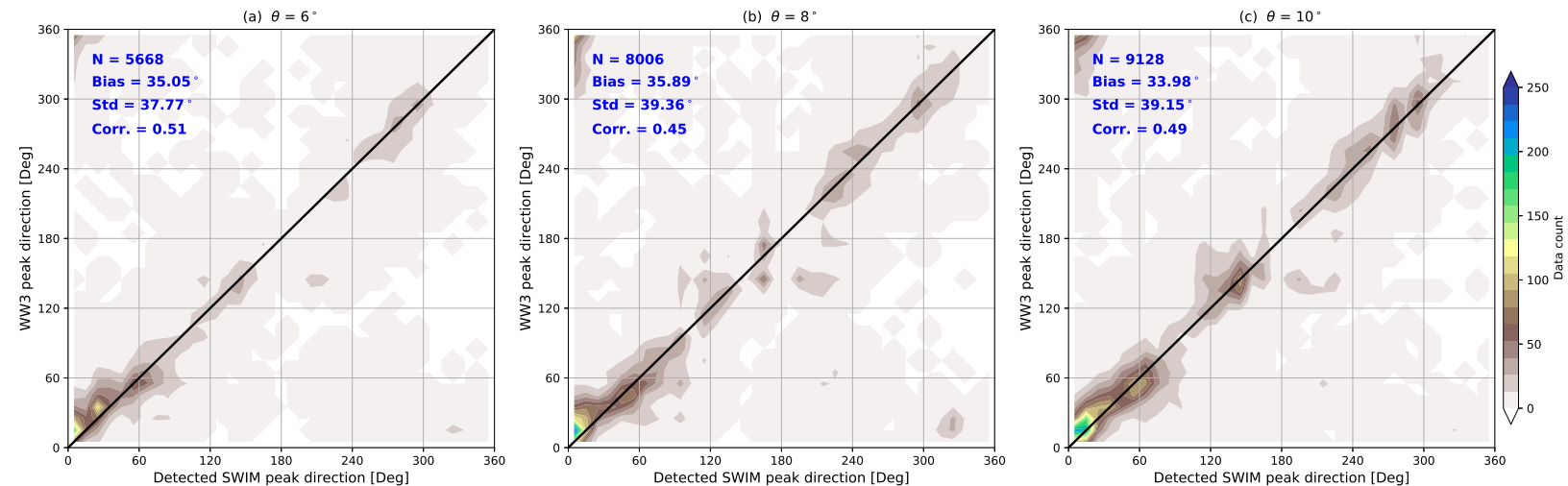

Fig. 9. Comparison of SWIM resolved wave peak direction with respect to the collocated WW3 wave peak direction for (a) $6^{\circ}$, (b) $8^{\circ}$ and (c) $10^{\circ}$ beams. 'N' annotated in each plot denotes the total number of valid peak pairs. Colors are the data count with directional bins of $10^{\circ}$ for both axes. Result metrics are given on the upper left corner of each plot.

the true wave direction.

\section{PRELIMINARY RESULTS OF WAVE DIRECTION AMBIGUITY REMOVAL}

The $R T_{P}$ in Fig. 7 illustrates that the fluctuation spectrum at the upwave configuration is indeed larger than its ambiguous counterpart at downwave direction. This characteristics agrees well with the trend of tilt MTF as reported in [28] that it has larger value in the upwave configuration. From the practical point of view, the observed fluctuation spectrum upto-downwave asymmetry can be readily utilized to help resolve the inherent $180^{\circ}$ wave direction ambiguity. As presented in Fig. 7, the downwave direction generally corresponds to the part with lower spectral values, which will be taken as the true wave direction (waves travelling to). This notation is consistent with the wave direction definition of traveling to in the WW3 products.

In this section, we focus on the wave direction ambiguity removal based on the up-to-downwave asymmetry of the fluctuation spectra. Fig. 9 displays the identified direction comparison between the SWIM detection results and the collocated WW3 wave spectra for the three spectral beams. It should be noted that the wave slope spectrum is utilized to extract the reference wave peaks. Since the fluctuation spectrum is utilized to identify the spectral peaks here, the spectral peak wavelengths might slightly differ with the reference wave peaks derived from the WW3 wave slope spectrum due to the impact of impulse response function and speckle noise spectrum. Yet the potential of such proposed algorithm is central to this study, we carried out the comparison between 
TABLE II

METRICS OF AMBIGUITY REMOVAL.

\begin{tabular}{|c|c|c|c|c|c|c|c|c|c|c|}
\hline \multirow{2}{*}{$\operatorname{INC}\left[{ }^{\circ}\right]$} & \multicolumn{3}{|c|}{ Total } & \multicolumn{3}{|c|}{$\mathrm{U} 10<15 \mathrm{~m} / \mathrm{s}$} & \multicolumn{3}{|c|}{$\mathrm{U} 10>15 \mathrm{~m} / \mathrm{s}$} & \multirow{2}{*}{ corr } \\
\hline & $\mathrm{N}$ & Bias & STD & $\mathrm{N}$ & Bias & STD & $\mathrm{N}$ & Bias & STD & \\
\hline 6 & 6442 & $34.99^{\circ}$ & $37.69^{\circ}$ & 6195 & $34.28^{\circ}$ & $37.05^{\circ}$ & 247 & $52.83^{\circ}$ & $47.99^{\circ}$ & 0.51 \\
\hline 8 & 8779 & $35.42^{\circ}$ & $38.90^{\circ}$ & 8543 & $34.94^{\circ}$ & $38.47^{\circ}$ & 236 & $52.84^{\circ}$ & $48.88^{\circ}$ & 0.46 \\
\hline 10 & 8978 & $33.86^{\circ}$ & $39.25^{\circ}$ & 8798 & $33.40^{\circ}$ & $38.74^{\circ}$ & 180 & $56.40^{\circ}$ & $54.64^{\circ}$ & 0.50 \\
\hline
\end{tabular}

these two datasets for simplicity.

As shown in Fig. 9, the extracted SWIM peak direction is in good alignment with the reference WW3 peak direction for all three spectral beams. Acquisitions from both ascending pass and descending pass are included in the analyses, thus the detected SWIM wave directions span in the entire range of $\left[0^{\circ}, 360^{\circ}\right]$. It should be reminded that the direction comparison demonstrated here is based on the spectral distance criteria of 3 for the peak-to-peak association and the angular sectors within $\pm 15^{\circ}$ relative to the track direction are masked out. The $10^{\circ}$ beams in Fig. 9(c) shows relatively better performance with the smallest direction bias of $33.86^{\circ}$ and the standard deviation of $39.25^{\circ}$. The $6^{\circ}$ beam shows comparable biases and standard deviation with the $8^{\circ}$ beam. The better performance of $10^{\circ}$ for ambiguity removal is expected due to the tight distribution of the $R T_{P}$ in Fig. 7. This is consistent with the previously reported results that the $10^{\circ}$ is mostly suitable for wave detection [18], [16]. While the correlation coefficient is sort of low, being $0.51,0.46$ and 0.50 for $6^{\circ}, 8^{\circ}$ and $10^{\circ}$, respectively. Combination of the three spectral beams is promising to improve the wave spectral inversion.

Some outliers are yet evident, which reduces the performance of the direction ambiguity removal based on the fluctuation spectrum asymmetry (scatter plot not shown here). For the $6^{\circ}$ beam in Fig. 9(a), a cluster of outliers appears at the position corresponding to the detected SWIM wave direction of $210^{\circ}$ and the WW3 wave direction of $150^{\circ} / 60^{\circ}$. The misjudgment of SWIM results can be traced back to the wider spread of $R T_{P}$ across the horizontal curve of one around the true wave direction of $60^{\circ}$ in Fig. 7. Note that the up- and downwave observations are usually $150 \mathrm{~km}$ apart, the assumption that the illuminated wave fields are consistent throughout might not hold in some case. This shall introduce extra errors in the ambiguity removal. In addition, the large scatter around $0^{\circ}$ and $360^{\circ}$ wave direction is because of the mask close to the track direction. The other wave directions are much cleaner owing to the tight distribution of $R T_{P}$. Similar cluster of outliers is observed for the other two beams of $8^{\circ}$ and $10^{\circ}$ in Fig. 7(b) and (c) as well.

In addition, the performance of direction ambiguity removal for two wind speed groups (separated by $15 \mathrm{~m} / \mathrm{s}$ ) is presented in Table. II. By comparison, the number of data points with the collocated wind speed higher than $15 \mathrm{~m} / \mathrm{s}$ is relatively small, accounting for only $35 \%$ of the total dataset. Both the direction bias and standard deviation for the high winds group show degraded results by approximately $20^{\circ}$. As a matter of fact, such decreasing performance is subject to a couple of possible reasons. For example, we have assumed that the ocean waves being measured remain unchanged over one entire rotation of SWIM beams (about $180 \mathrm{~km} \times 180 \mathrm{~km}$ ), which might not be true under rapidly changing high winds. Also, the capability of WW3 to accurately resolve the wave spectrum under high winds also needs further evaluation [35]. Dedicated studies to propose new algorithm typical of high winds are of great interests and necessity.

\section{DISCUSSION AND CONCLUSION}

The concept of a conical scanning real aperture radar operating at near-nadir incidence angles has been proposed and validated using the airborne instruments for decades. With the successful launch of COFSAT, such observations of ocean waves at the global scale are available for the first time ever. Despite the constant effort to update the operational data processing algorithms, the inherent $180^{\circ}$ wave direction ambiguity yet hinders some geophysical applications using the SWIM measurements. In this paper, the up-to-downwave asymmetry of the estimated fluctuation spectrum is first documented. It is worth noting that the fluctuation spectrum over the angular sector of $\pm 15^{\circ}$ relative to the track direction has been excluded in this analysis due to the high level of speckle noise in this direction. Outside this sector, the mean speckle noise spectrum is subtracted for each incidence beam. The ratio of fluctuation spectrum peaks within $\left[0^{\circ}, 180^{\circ}\right]$ to its ambiguous counterpart within $\left[180^{\circ}, 360^{\circ}\right]$ shows distinct behavior relative to the true wave direction. It can be summarized that the fluctuation spectrum is significantly larger in the upwave than in the downwave direction as shown in Fig. 7. The preliminary results to resolve the wave direction ambiguity in terms of this up-to-downwave asymmetry are promising with relatively better performance of the $10^{\circ}$ beam.

This documented asymmetry is consistent with the simulation based on the theoretical simulations with the input of tilt MTF and wave field. At the near-nadir incidence angles, the linear approximation theory relates the modulation spectrum to the wave spectrum via the so-called tilt MTF, which is defined by the change rate of backscattering relative to the incidence angles. Since the wave spectrum can be considered unchanged within the footprint on each side of the SWIM track, under the linear theory, a larger spectrum in the upwave with respect to the downwave can only be explained by a larger MTF in that direction. In [28], the authors analyzed the tilt MTF from an analysis of the backscattering coefficients provided by the Global Precipitation Mission (GPM). They found that the tilt MTF in Ku-Band and at $10^{\circ}$ incidence is indeed 5 to $10 \%$ larger in upwind than in downwind direction for winds between 10 and $15 \mathrm{~m} / \mathrm{s}$. Hence, according 
to the results of [28], the modulation spectrum in downwave should be smaller than that in upwave. This agrees well with the analyses results presented in Fig. 7. The quantitative comparison of such asymmetry yet requires further efforts. For example, the nonlinear range bunching is usually neglected in the state-of-the-art SWIM simulators, which might modify the magnitude of up-to-downwave asymmetry of the simulated fluctuation spectrum. Other factors including the high-order hydrodynamic modulation and the instrumental performance needs more investigations as well. In any case, this consistent asymmetry shall provide valuable data reference to complete our understanding on SWIM measurement principles. Note that the ambiguity removal in terms of the up-to-downwave asymmetry is based on the assumption that the wave fields do not experience significant variation within one entire rotation of $360^{\circ}$, which is roughly equivalent to $170 \mathrm{~km} \times 170 \mathrm{~km}$ in space. Another factor to be considered for the operational implementation is the wind-swell direction, which might result in opposite direction bias.

In addition to the application for direction ambiguity removal, the skewed fluctuation spectrum between upwave and downwave shall be further employed to refine the wave inversion. With the ambiguity resolved and the along-track noise issue corrected in the latest available products, the derived two-dimensional wave spectra shall actually help to fully characterize the sea state conditions across the globe. More oceanographic studies will benefit from the simultaneous measurements of ocean waves by SWIM and wind field by the scatterometer aboard CFOSAT. For example, the wind-wave interaction can be possibly quantified as well as the local airsea interaction. Wave in the sea ice is also an promising aspect to investigate, just to name a few.

The preliminary results of ambiguity removal by utilizing the up-to-downwave asymmetry demonstrated in this study are affirmative for the future operational implementation. Though, a more thorough algorithm needs to be devised for quantification of up-to-downwave asymmetry of wave partitions in addition to the local peaks. The applicability of such algorithm at relatively high winds also needs further evaluation. With these points properly addressed, this algorithm shall greatly help refine the wave inversion scheme as well as enhance the usage of SWIM observations in oceanographic related studies.

\section{ACKNOWLEDGMENT}

We would like to acknowledge the CNSA and CNES for providing the CFOSAT/SWIM Level-1A and Level1B products, which can be publicly downloaded via https:/www.aviso.altimetry.fr/en/missions/current-missions/ cfosat/access-to-data.html. The ECMWF forecast winds are obtained in the framework of Sentinel-1 Mission Performance and are publicly available (ecmwf.int). WaveWatch III wave spectra are produced at Ifremer/LOPS within the framework of CFOSAT mission. All CFOSAT products are provided by the courtesy of CNSA and CNES.

\section{REFERENCES}

[1] Naoto Ebuchi, Yoshiaki Toba, and Hiroshi Kawamura, "Statistical study on the local equilibrium between wind and wind waves by using data from ocean data buoy stations," Journal of Oceanography, vol. 48, no. 1, pp. 77-92, Apr 1992.

[2] Weimin Huang, Shicai Wu, E. Gill, Biyang Wen, and Jiechang Hou, "Hf radar wave and wind measurement over the eastern china sea," IEEE Transactions on Geoscience and Remote Sensing, vol. 40, no. 9, pp. 1950-1955, 2002.

[3] Jian-Guo Li and Andrew Saulter, "Assessment of the updated envisat asar ocean surface wave spectra with buoy and altimeter data," Remote Sensing of Environment, vol. 126, pp. 72 - 83, 2012.

[4] J. An, W. Huang, and E. W. Gill, "A self-adaptive wavelet-based algorithm for wave measurement using nautical radar," IEEE Transactions on Geoscience and Remote Sensing, vol. 53, no. 1, pp. 567-577, 2015.

[5] J. Horstmann, H. Schiller, J. Schulz-Stellenfleth, and S. Lehner, "Global wind speed retrieval from sar," IEEE Transactions on Geoscience and Remote Sensing, vol. 41, no. 10, pp. 2277-2286, Oct 2003.

[6] Hui Lin, Qing Xu, and Quanan Zheng, "An overview on sar measurements of sea surface wind," Progress in Natural Science, vol. 18, no. 8, pp. $913-919,2008$.

[7] F. Collard, F. Ardhuin, and B. Chapron, "Extraction of coastal ocean wave fields from sar images," IEEE Journal of Oceanic Engineering, vol. 30, no. 3, pp. 526-533, July 2005.

[8] Werner R. Alpers, Duncan B. Ross, and Clifford L. Rufenach, "On the detectability of ocean surface waves by real and synthetic aperture radar," Journal of Geophysical Research: Oceans, vol. 86, no. C7, pp. 6481-6498, 1981.

[9] W. R. Alpers and C. Bruening, "On the relative importance of motionrelated contributions to the sar imaging mechanism of ocean surface waves," IEEE Transactions on Geoscience and Remote Sensing, vol. GE-24, no. 6, pp. 873-885, Nov 1986.

[10] Klaus Hasselmann and Susanne Hasselmann, "On the nonlinear mapping of an ocean wave spectrum into a synthetic aperture radar image spectrum and its inversion," Journal of Geophysical Research: Oceans, vol. 96, no. C6, pp. 10713-10729, 1991.

[11] Harald E. Krogstad, "A simple derivation of hasselmann's nonlinear ocean-synthetic aperture radar transform," Journal of Geophysical Research: Oceans, vol. 97, no. C2, pp. 2421-2425, 1992.

[12] Frederick C. Jackson, W. Travis Walton, and Paul L. Baker, "Aircraft and satellite measurement of ocean wave directional spectra using scanningbeam microwave radars," Journal of Geophysical Research: Oceans, vol. 90, no. C1, pp. 987-1004, 1985.

[13] Frederick C. Jackson, W. Travis Walton, and Chich Y. Peng, "A comparison of in situ and airborne radar observations of ocean wave directionality," Journal of Geophysical Research: Oceans, vol. 90, no. C1, pp. 1005-1018, 1985.

[14] D. Hauser, G. Caudal, G. . Rijckenberg, D. Vidal-Madjar, G. Laurent, and P. Lancelin, "Ressac: a new airborne $\mathrm{fm} / \mathrm{cw}$ radar ocean wave spectrometer," IEEE Transactions on Geoscience and Remote Sensing, vol. 30, no. 5, pp. 981-995, 1992.

[15] Danièle Hauser, Elbatoul Soussi, Eric Thouvenot, and Laurent Rey, "Swimsat: A real-aperture radar to measure directional spectra of ocean waves from space-main characteristics and performance simulation," Journal of Atmospheric and Oceanic Technology, vol. 18, no. 3, pp. 421-437, 2001.

[16] Danièle Hauser, C. Tison, T. Amiot, L. Delaye, N. Corcoral, and P. Castillan, "Swim: The first spaceborne wave scatterometer," IEEE Transactions on Geoscience and Remote Sensing, vol. 55, no. 5, pp. 3000-3014, 2017.

[17] J. Liu, W. Lin, X. Dong, S. Lang, R. Yun, D. Zhu, K. Zhang, C. Sun, B. Mu, J. Ma, Y. He, Z. Wang, X. Li, X. Zhao, and X. Jiang, "First results from the rotating fan beam scatterometer onboard cfosat," IEEE Transactions on Geoscience and Remote Sensing, pp. 1-14, accepted, 2020.

[18] D. Hauser, C. Tourain, L. Hermozo, D. Alraddawi, L. Aouf, B. Chapron, A. Dalphinet, L. Delaye, M. Dalila, E. Dormy, F. Gouillon, V. Gressani, A. Grouazel, G. Guitton, R. Husson, A. Mironov, A. Mouche, A. Ollivier, L. Oruba, F. Piras, R. Rodriguez Suquet, P. Schippers, C. Tison, and N. Tran, "New observations from the swim radar on-board cfosat: Instrument validation and ocean wave measurement assessment," IEEE Transactions on Geoscience and Remote Sensing, vol. 59, no. 1, pp. 5-26, 2021.

[19] Kirsty E. Hanley, Stephen E. Belcher, and Peter P. Sullivan, "A global climatology of wind-wave interaction," Journal of Physical Oceanography, vol. 40, no. 6, pp. 1263-1282, 2010.

[20] L. Aouf, A. Dalphinet, D. Hauser, L. Delaye, C. Tison, B. Chapron, L. Hermozo, and C. Tourain, "On the assimilation of cfosat wave data in the wave model mfwam : Verification phase," in Proceedings of the 
2019 IEEE International Geoscience and Remote Sensing Symposium, Yokohama, Japan, 2019, p. ...

[21] L. Aouf, D. Hauser, B. Chapron, A. Toffoli, C. Tourrain, and C. Peureux, "New directional wave satellite observations : Towards improved wave forecasts and climate description in southern ocean," Geophysical Research Letters, vol. n/a, no. n/a, pp. e2020GL091187, 2021, e2020GL091187 2020GL091187.

[22] Gaspar R Valenzuela, "Theories for the interaction of electromagnetic and oceanic waves - A review," Boundary-Layer Meteorology, vol. 13, no. 1, pp. 61-85, jan 1978.

[23] Frederick C. Jackson, "An analysis of short pulse and dual frequency radar techniques for measuring ocean wave spectra from satellites," Radio Science, vol. 16, no. 6, pp. 1385-1400, 1981.

[24] C. Cox and W. Munk, "Statistics of the sea surface derived from sun glitter," Journal of Marine Research, vol. 13, no. 2, pp. 198-227, 1954.

[25] Michael H. Freilich and Barry A. Vanhoff, "The relationship between winds, surface roughness, and radar backscatter at low incidence angles from trmm precipitation radar measurements," Journal of Atmospheric and Oceanic Technology, vol. 20, no. 4, pp. 549-562, 2003.

[26] William J. Plant, "A new interpretation of sea-surface slope probability density functions," Journal of Geophysical Research: Oceans, vol. 108, no. C9, 2003.

[27] Ping Chen, Gang Zheng, Danièle Hauser, and Fei Xu, "Quasi-gaussian probability density function of sea wave slopes from near nadir ku-band radar observations," Remote Sensing of Environment, vol. 217, pp. 86 100,2018

[28] Victor Gressani, Frédéric Nouguier, and Alexis Aurélien Mouche, "Wave spectrometer tilt modulation transfert function using near-nadir kuand ka-band gpm radar measurements," IGARSS 2018 - 2018 IEEE International Geoscience and Remote Sensing Symposium, pp. 41074110,2018

[29] Tison Céline and Hauser Daniele, "Swim products users guide: Product description and algorithm theoretical baseline description," Tech. Rep., Centre National d'Etudes Spatiales, 2019.

[30] Fabrice Ardhuin, Erick Rogers, Alexander V. Babanin, Jean-François Filipot, Rudy Magne, Aaron Roland, Andre van der Westhuysen, Pierre Queffeulou, Jean-Michel Lefevre, Lotfi Aouf, and et al., "Semiempirical dissipation source functions for ocean waves. part i: Definition, calibration, and validation," Journal of Physical Oceanography, vol. 40, no. 9, pp. 1917-1941, Sep 2010.

[31] Justin E. Stopa, Fabrice Ardhuin, Alexander Babanin, and Stefan Zieger, "Comparison and validation of physical wave parameterizations in spectral wave models," Ocean Modelling, vol. 103, pp. 2 - 17, 2016.

[32] Fawwaz T. Ulaby, Richard K. Moore, and Adrian K. Fung, Microwave Remote Sensing: Active and Passive Volume II, Reading, Mass. : Addison-Wesley, 1982.

[33] Frederick C. Jackson, "The radar ocean-wave spectrometer," in 1986 Johns Hopkins APL Technical Digest (ISSN 0270-5214), Jan.-Mar. 1987, vol. 8, pp. 116-127.

[34] Romain Husson, Development and validation of a global observationbased swell model using wave mode operating Synthetic Aperture Radar, Ph.D. thesis, UNIVERSITE DE BRETAGNE OCCIDENTALE, 102012.

[35] Yalin Fan and W. Erick Rogers, "Drag coefficient comparisons between observed and model simulated directional wave spectra under hurricane conditions," Ocean Modelling, vol. 102, pp. 1-13, 2016.

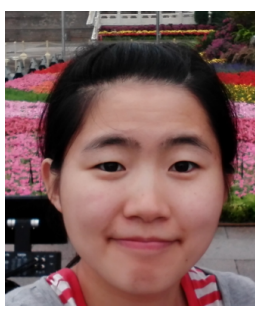

Huimin Li received the B.S. and M.S. degrees in ocean information detection from the Ocean University of China, Qingdao, China, in 2013 and 2015, respectively, and the Ph.D. degree from IMT-Atlantique/LOPS-SIAM, Institut Français de Recherche pour l'Exploitation de la Mer (IFREMER), Brest, France, in 2019. She joined the School of Marine Sciences, Nanjing University of Information Science and Technology since July 2019.

Her research interests include better understanding of synthetic aperture radar (SAR) imaging mechanism over ocean waves and the analysis of Sentinel-1 SAR images to obtain simultaneous observations of sea surface wind, ocean wave field, and oceanic current.

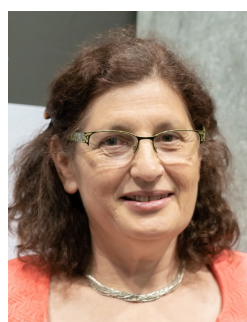

satellite.
Danièle Hauser is Senior Scientist at CNRS (Centre National de la Recherche Scientifique) and develops her research activity at LATMOS (Laboratoire Atmosphère, Observations Spatiales). She received a $\mathrm{PhD}$ Thesis in meteorology in 1980 and a State Thesis in Physics in 1989. She is working for more than 30 years on microwave observations of the ocean surface (surface wind, waves, salinity) and air/sea interactions studies. She is presently Principal Investigator of the CFOSAT mission dedicated to the global measurement of wind and waves from

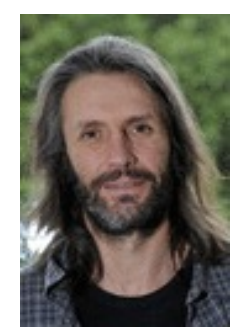

Bertrand Chapron received the Ph.D. degree in fluid mechanics from Aix-Marseille University, Marseille, France, in 1988

$\mathrm{He}$ is currently a Senior Research Scientist with the Laboratoire d'Océanographie Physique Spatile ,Institut Francais de Recherche pour l'Exploitation de la Mer, Plouzane, France. He has been the Co-Investigator or Principal Investigator in several ESA (ENVISAT RA2, ASAR, and SMOS), NASA, and CNES (TOPEX and JASON) projects. He contributed to the development of several algorithms for geophysical parameter retrieval from altimeters, radiometers, or SAR. He is co-responsible for the ENVISAT ASAR-Wave Mode algorithms and scientific preparation for the ENVISAT and S1 wind, wave, and current. Recently, he collaborated in studies that demonstrated the high potential of radiometers for wind retrieval in extreme conditions and the possibility of doing directional ocean waves spectrum from Sentinel-2. He has authored over 80 publications in refereed journals in applied mathematics, physical oceanography (upper ocean dynamics), and electromagnetic wave theory and its application to ocean surface remote sensing.

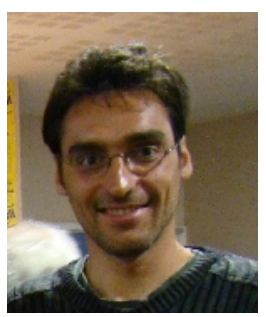

Frederic Nouguier received the "Agregation" and the M.S. degrees in applied physics from the Ecole Normale Superieure de Cachan, France, the M.S. degree in physical methods for remote sensing from the University of Paris-Diderot, Paris and the Ph.D. degree in physics from the University of Marseille, France, in 2009. He is now a Research Scientist at the Laboratoire d'Océanographie Physique et Spatiale (LOPS), IFREMER, Plouzane, France. He has experience in applied mathematics, physical oceanography, and electromagnetic wave theory and its application to ocean remote sensing.

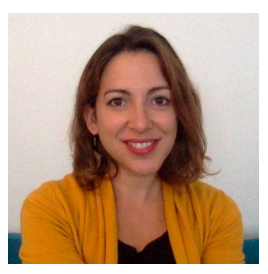

Patricia Schippers received a master degree in Engineering in Physics of Université Libre de Bruxelles, Belgium, in 2004, and the Doctor of Astrophysics degree, Université Paul Sabatier, Toulouse, France, in 2009.

Her skills include statistical and scientific analysis of geophysical and planetary data, inversion algorithm implementation for satellite data. She is currently working as a research engineer with ACRIST as a scientific support on the Chinese-French oceanography mission CFOSAT for the LATMOS laboratory. She is involved in the specification and validation of the SWIM radar data processing chain, quality control and performance of data products, anomaly investigation and scientific analysis of high-level products (wave spectra and parameters). 


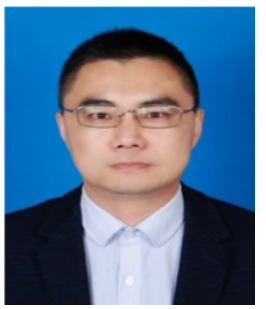

Biao Zhang (M'08-SM'16) received the B.S. degree in surveying and mapping engineering from the China University of Petroleum, Dongying, China, in 2003, and the Ph.D. degree in physical oceanography from the Institute of Oceanology, Chinese Academy of Sciences, Qingdao, China, in 2008.

$\mathrm{He}$ had been with the Bedford Institute of Oceanography, Canada, from 2008 to 2011, where he was a Postdoctoral Fellow and involved in developing synthetic aperture radar ocean surface wave and wind retrieval algorithms and products. $\mathrm{He}$ is currently a Professor with the School of Marine Sciences, Nanjing University of Information Science and Technology (NUIST). His research interests include satellite remote sensing of marine dynamic environment and tropical cyclone, air-sea interaction under extreme weather conditions, Arctic sea ice monitoring by active and passive microwave sensors and radar constellation mission.

Dr. Zhang was a recipient of the Visiting Fellow Scholarship of Natural Sciences and Engineering Research Council of Canada. He received the first award of Science and Technology from Jiangsu Province in 2017, and the first award of Ocean Science and Technology from the State Oceanic Administration of China, and the second award of Natural Science from the Ministry of Education of China in 2014. He was a recipient of the Outstanding Young Scientist Award by National Science Foundation of China in 2016. He was selected "six talent peaks" of Jiangsu Province in 2018.

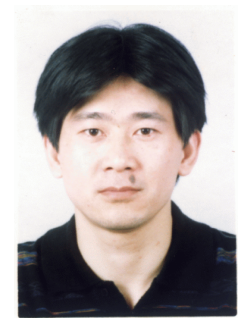

Jingsong Yang received the B.S. degree in physics and the M.S. degree in theoretical physics from Zhejiang University, Hangzhou, China, in 1990 and 1996, respectively, and the Ph.D. degree in Physical Oceanography from Ocean University of China, Qingdao, China, in 2001.

$\mathrm{He}$ is with the Second Institute of Oceanography (SIO), Ministry of Natural Resources (MNR), Hangzhou, China, where he is the Head of the Microwave Marine Remote Sensing, State Key Laboratory of Satellite Ocean Environment Dynamics.

$\mathrm{He}$ is also an Adjunct Professor and a Doctoral Supervisor with Zhejiang University, Shanghai Jiao Tong University and Hohai University. He is also a senior member of Southern Marine Science and Engineering Guangdong Laboratory (Zhuhai). He has more than 20 years of experience in microwave marine remote sensing. He has been a Principal Investigator and a Participant of more than 20 research projects and published more than 100 scientific articles in peer-reviewed journals and international conference proceedings. His research interests include microwave marine remote sensing, data fusion, image processing, and satellite oceanography.

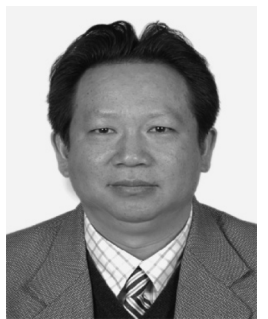

Yijun He (Member, IEEE) received the B.S. degree in physics from Hunan Normal University, Changsha, China, in 1985, the M.S. degree in applied physics from the Xidian University, Xi'an, China, in 1990, and the Ph.D. degree in microwave theory and technology from the Southeast University, Nanjing, China, in 1993.,He was with the Key Laboratory of Ocean Circulation and Waves, Institute of Oceanology, Chinese Academy of Sciences, Qingdao, China, where he was the Head of the Remote Sensing Group and a Senior Scientist of satellite oceanography from 1996 to 2011. He was a Post-Doctoral Fellow with the Ocean Remote Sensing Institute, Ocean University of China, Qingdao, from 1993 to 1996. He was a Visiting Scientist with the University of Hamburg, Hamburg, Germany, with the Bedford Institute of Oceanography, Dartmouth, NS, Canada, and also with the University of Delaware, Newark, DE, USA.

$\mathrm{He}$ is currently a Professor of ocean remote sensing and the Dean of the School of Marine Sciences, Nanjing University of Information Science and Technology (NUIST), Nanjing. His research interests include ocean waves, sea surface wind speed, and other ocean surface features related to remote sensing by full polarization synthetic aperture radar and other microwave radars at low incidence angle, air-sea gas exchange using microwave remote sensing and numerical models, sea surface scattering of electromagnetic waves, and mesoscale and submesoscale ocean dynamics. 